\title{
Synthesis of the Tetracyclic Core of Daphnilactone B-Type and Yuzurimine- Type Alkaloids
}

2011

Vol. 13, No. 23

6204-6207

\section{Guillaume Bélanger, ${ }^{\star}$ Jonathan Boudreault, and François Lévesque}

Département de Chimie, Université de Sherbrooke, 2500 Boulevard de l'Université, Sherbrooke, Québec J1K 2R1, Canada

Guillaume.Belanger@USherbrooke.ca

Received September 29, 2011

\section{ABSTRACT}

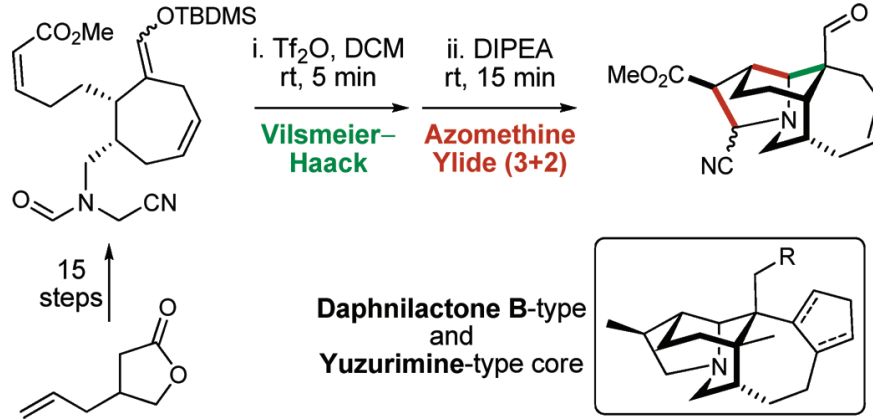

The core of daphnilactone B-type and yuzurimine-type alkaloids was synthesized in only 16 steps from a known $\beta$-allyl- $\gamma$-butyrolactone. The key sequence of Vilsmeier-Haack cyclization and intramolecular azomethine ylide cycloaddition allowed the construction of, in a single step, three of the five rings common to all alkaloids found in both of these classes with perfect chemocontrol.

Daphniphyllum alkaloids form an extremely rich class of more than 220 natural products. ${ }^{1}$ They exhibit a wide range of biological activity and present complex architectures that required ingenious approaches to achieve their syntheses. Daphnane alkaloids are classified into 16 types, based on their polycyclic skeletons. ${ }^{2}$ Five of them, sharing a closely related framework, are represented in Figure 1.

Although many elegant and highly efficient syntheses have been reported for several alkaloids found in the bukkittingine, ${ }^{3}$ secodaphniphylline, ${ }^{4}$ and daphniphylline ${ }^{5}$ classes, no synthesis of any alkaloids in the daphnilactone $\mathrm{B}$ and the yuzurimine classes has been published. Despite

(1) Yang, T.-Q.; Dia, Y.-T.; Hea, H.-P.; Zhanga, Q.; Zhanga, Y.; Hao, X.-J. Helv. Chim. Acta 2011, 397.

(2) Kobayashi, J.; Kubota, T. Nat. Prod. Rep. 2009, 26, 936.

(3) For $( \pm$ )-bukittingine, see: Heathcock, C. H.; Stafford, J. A.; Clark, D. L. J. Org. Chem. 1992, 57, 2575.

(4) For methyl ( \pm )-homosecodaphniphyllate, see: (a) Ruggeri, R. B.; Hansen, M. M.; Heathcock, C. H. J. Am. Chem. Soc. 1988, 110, 8734. For (-)-secodaphniphylline, see:(b) Stafford, J. A.; Heathcock, C. H. J. Org. Chem. 1990, 55, 5433 .

(5) For methyl ( \pm )-homodaphniphyllate, see: (a) Heathcock, C. H.; Davidsen, S. K.; Mills, S.; Sanner, M. A. J. Am. Chem. Soc. 1986, 108, 5650. For codaphniphylline, see:(b) Heathcock, C. H.; Kath, J. C.; Ruggeri, R. B. J. Org. Chem. 1995, 60, 1120. the elucidation of the structures of several daphnilactone $\mathrm{B}^{6}$ and yuzurimine ${ }^{7}$ daphnanes reported up to 35 years ago, ${ }^{6 b, 7 a}$ the synthesis of these alkaloids continues to be a particularly difficult unmet challenge.

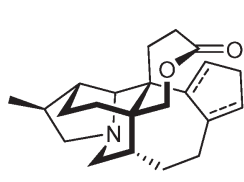

Daphnilactone B-type

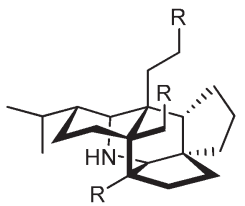

Secodaphniphylline-type

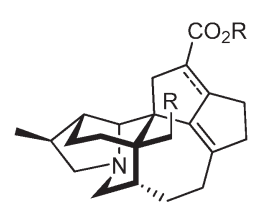

Yuzurimine-type

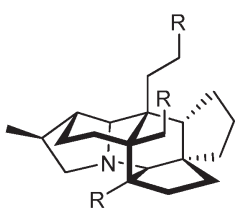

Bukittinggine-type 
We became interested in daphnilactone B-type and yuzurimine-type alkaloids as excellent targets to demonstrate the applicability of the sequence of the intramolecular Vilsmeier-Haack reaction and azomethine ylide cycloaddition we developed recently. ${ }^{8}$ This strategy would allow the construction of, in a single step, three of the five rings common to all alkaloids found in both of these classes. Our retrosynthetic analysis of target product $\mathbf{1}$ starts with the formation of the pyrrolidine ring using a stabilized azomethine ylide $(3+2)$ cycloaddition (Scheme 1$)$. This ylide intermediate $\mathbf{2}$ would be generated from the deprotonation of an iminium ion obtained from a Vilsmeier-Haack cyclization initiated by the activation of formamide 3. A ring closing metathesis would serve to form the cycloheptene ring of $\mathbf{3}$, while a Still-Gennari olefination would generate the cis unsaturated ester. The cis isomer would set the right configuration at $\mathrm{C}_{8}$ in the tetracyclic product $\mathbf{1}$ for further reduction to the methyl in the natural products. The $\alpha$-allylaldehyde moiety in $\mathbf{4}$ would come from a Claisen rearrangement of an allyl enol ether, and the amido-nitrile portion would be the product of functional group manipulation from bromide 5. The latter would be derived from $\mathbf{6}$ by alkylation $\alpha$ to the carbonyl and further transformations of the lactone.

While no completed synthesis of alkaloids in the daphnilactone $\mathrm{B}$ and yuzurimine classes has been reported so far, two research groups published their approaches to the construction of the core of these daphnanes. Denmark's group took advantage of their well established cascade of intramolecular nitroalkene $(4+2)$ cycloaddition and nitronate $(3+2)$ cycloaddition to synthesize the bridged indolizidine core of these alkaloids, in an enantioenriched form (Scheme 2). ${ }^{9}$ The advanced intermediate 9 was thus prepared in 27 steps. Their approach even allowed for the very challenging formation of the two contiguous quaternary centers (cf. 9, $\mathrm{C}_{3}$ and $\mathrm{C}_{4}$ ) found in all alkaloids of these classes, although when compared to the natural alkaloids, product 9 contains an additional six-membered ring and the seven-membered ring is missing.

Earlier this year, the group of Coldham reported the condensation of bromoaldehyde $\mathbf{1 1}$ with ethyl glycinate, followed by intramolecular alkylation and deprotonation

(6) (a) For daphnilactone B, see: Sasaki, K.; Hirata, Y. Tetrahedron Lett. 1972, 13, 1891-1894. (b) For daphnezomine H and I, see: Morita, H.; Yoshida, N.; Kobayashi, J. Tetrahedron 2000, 2641. (c) For caldaphnidine C, see: Zhan, Z.-J.; Zhang, C.-R.; Yue, J.-M. Tetrahedron 2005, 11038.

(7) (a) For deoxyyuzurimine, see: Yamamura, S.; Terada, Y. Chem. Lett. 1976, 1381. (b) For daphnetidine D, see: Kubota, T.; Matsuno, Y.; Morita, H.; Shinzato, T.; Sekiguchid, M.; Kobayashi, J. Tetrahedron 2006, 4743. (c) For daphniglaucines J and K, see: Takatsu, H.; Morita, H.; Shenb, Y.-C.; Kobayashi, J. Tetrahedron 2004, 6279. (d) For daphcalycic acid, see: El Bitar, H.; Nguyen, V. H.; Gramain, A.; Sévenet, T.; Bodo, B. Tetrahedron Lett. 2004, 515. (e) For daphnezomine K, see ref $6 \mathrm{~b}$. (f) For caldaphnidine A, see ref $6 \mathrm{c}$.

(8) (a) Lévesque, F.; Bélanger, G. Org. Lett. 2008, 10, 4939. (b) Bélanger, G.; Darsigny, V.; Doré, M.; Lévesque, F. Org. Lett. 2010, 12, 1396.

(9) (a) Denmark, S. E.; Baiazitov, R. Y. J. Org. Chem. 2006, 71, 593. (b) Denmark, S. E.; Baiazitov, R. Y.; Nguyen, S. T. Tetrahedron 2009, 65, 6535 .

(10) Coldham, I.; Burrell, A. J. M.; Guerrand, H. D. S.; Oram, N. Org. Lett. 2011, 13, 1267.
Scheme 1. Retrosynthetic Analysis of Daphnilactone B-Type and Yuzurimine-Type Alkaloids
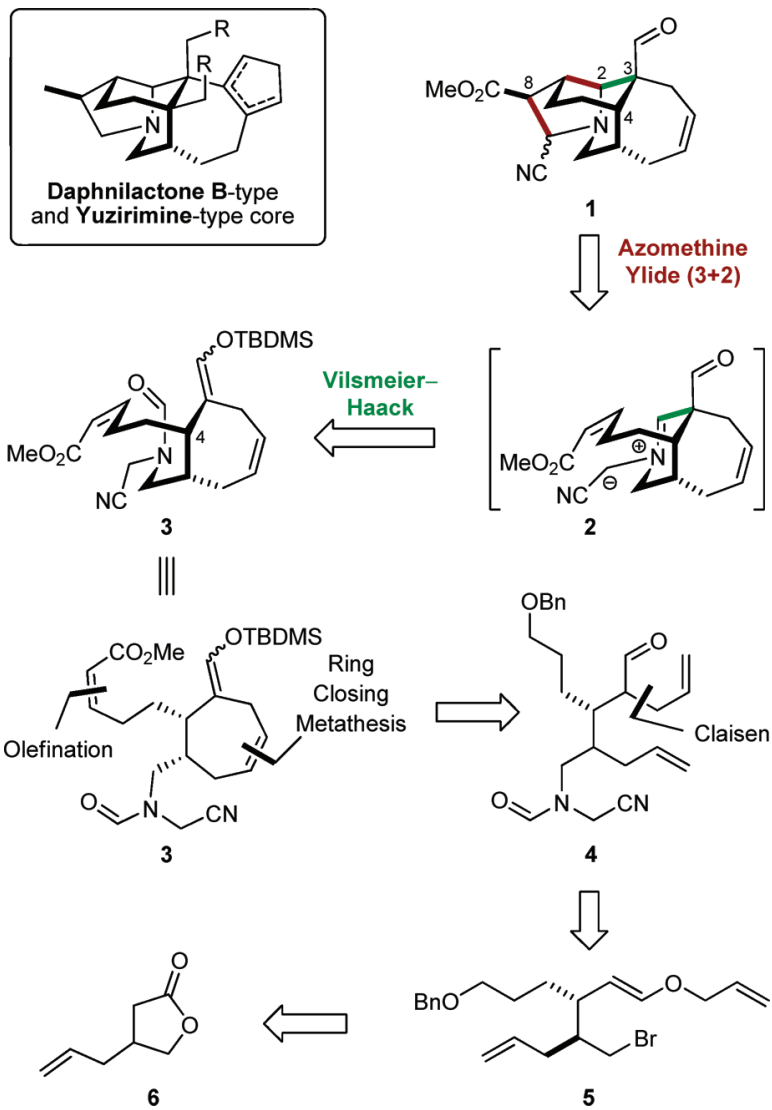

5

Scheme 2. Denmark's Approach
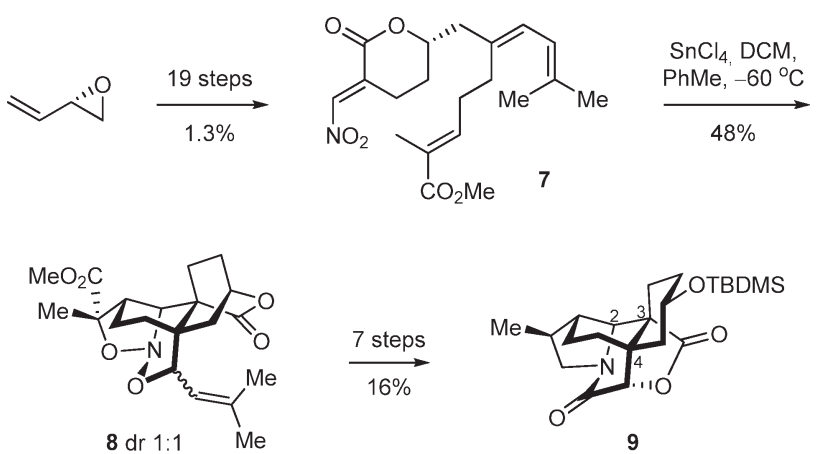

to generate a transient azomethine ylide that was trapped in an intramolecular cycloaddition (Scheme 3). ${ }^{10}$ The approach is very short (11 steps) to get to $\mathbf{1 2}$ that however lacks the seven-membered ring and the $\mathrm{C}_{4}$ quaternary center. $^{11}$

As detailed in Scheme 1, the synthetic strategy we put forward has the ultimate goal of setting for the first time all four cycles of the core of daphnilactone B and yuzurimine

(11) Our approach lacks the $\mathrm{C}_{4}$ quaternary center as well. However, the latter would be easily incorporated from alkylation on compound $\mathbf{6}$. 
Scheme 3. Coldham's Approach

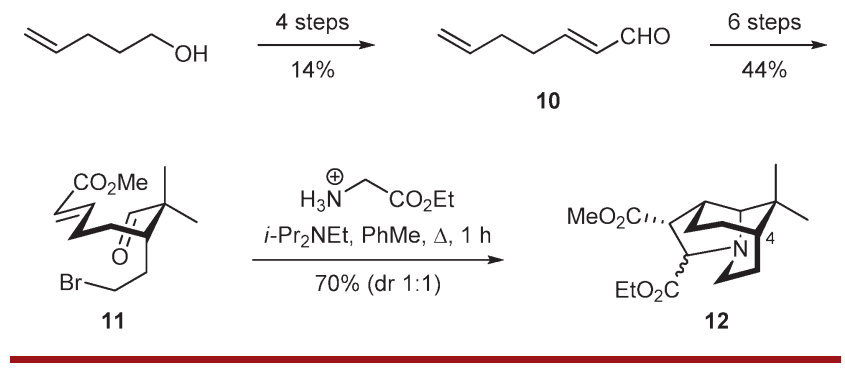

Scheme 4. Synthesis of the Key Step Precursor 3
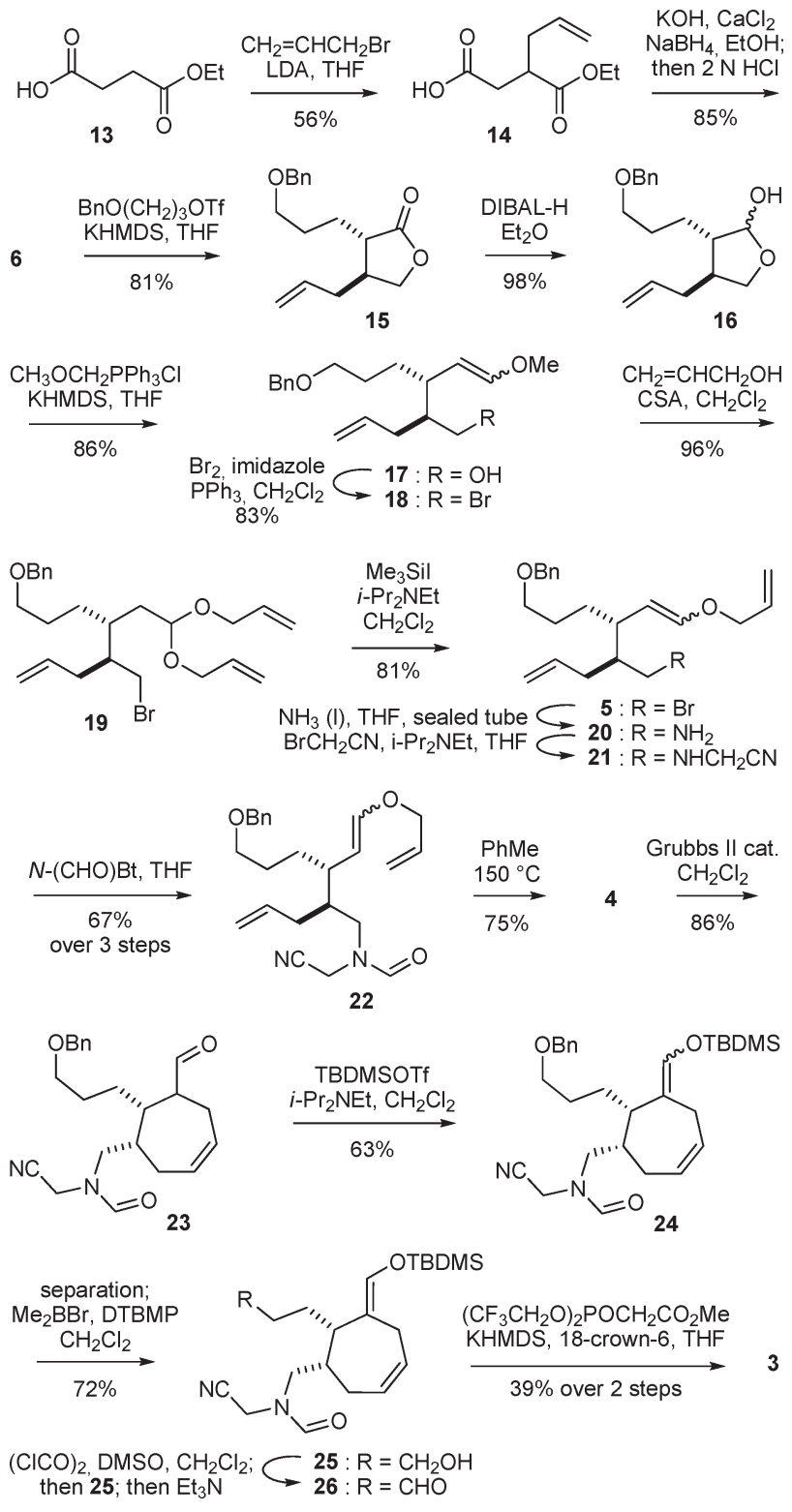

alkaloids in a diastereocontrolled and concise way. Our synthesis started with a double deprotonation and monoallylation of succinic acid monoethyl ester (13,
Scheme 4). ${ }^{12}$ Reduction of the ester in the presence of the carboxylic acid generated the corresponding hydroxyacid that spontaneously lactonized upon acidification of the reaction medium. ${ }^{13}$ Lactone 6 was alkylated to install the precursor of the dipolarophile required for the key step. ${ }^{14}$ The trans relative stereochemistry of $\mathbf{1 5}$ was established by $\mathrm{X}$-ray diffraction of crystalline $p$-bromobenzoate $\mathbf{2 8}$ obtained after catalytic hydrogenation of $\mathbf{1 5}$ and benzoylation of alcohol 27 (Scheme 5).

Lactone 15 was then reduced, and the resulting lactol 16 was olefinated to the methyl enol ether 17 (Scheme 4). After bromination of the latter, product $\mathbf{1 8}$ was treated with an excess of allyl alcohol to generate the diallyl acetal which was then reacted with TMSI $^{15}$ to eliminate allyl alcohol and furnish the allyl enol ether $\mathbf{5}$. The amido-nitrile moiety was readily installed from bromide $\mathbf{5}$ via nucleophilic displacement with ammonia, followed by mono$N$-alkylation using bromoacetonitrile and formylation. ${ }^{16}$ Intermediate 22 was heated to induce a Claisen [3,3]rearrangement, and the resulting $\alpha$-allylaldehyde was ring-closed using a second generation Grubbs catalyst in very good yield. ${ }^{17}$ Enolization of aldehyde 23 set the nucleophile required for the Vilsmeier-Haack cyclization in the key transformation. ${ }^{18}$

Scheme 5. Proof of Relative Stereochemistry on $\mathbf{1 5}$

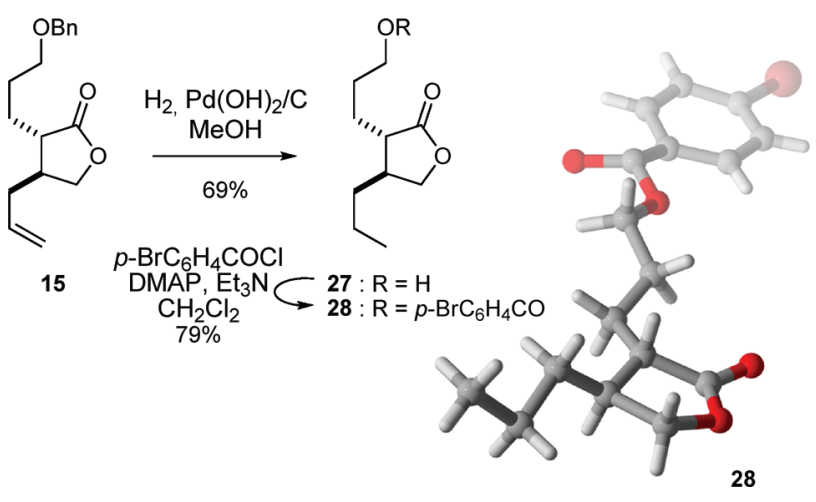

Removal of the benzyl group on $\mathbf{2 4}$ in the presence of the nitrile, the formamide, and the sensitive silyl enol ether was

(12) Berbmeier, S. C.; Ismail, K. A. Synthesis 2000, 10, 1369.

(13) Robin, J.-P.; Landais, Y. Tetrahedron 1992, 48, 819.

(14) (a) Baldoli, C.; Cerea, P.; Falciola, L.; Giannini, C.; Licandro, E.; Maiorana, S.; Mussini, P.; Perdicchia, D. J. Organomet. Chem. 2005, 690, 5777. (b) Uenishi, J.; Tatsumi, Y.; Kobayashi, N.; Yonemitsu, O. Tetrahedron Lett. 1995, 36, 5909.

(15) Kadota, I.; Takamura, H.; Sato, K ; Ohno, A ; Matsuda, K. Satake, M.; Yamamoto, Y. J. Am. Chem. Soc. 2003, 125, 11893.

(16) (a) Katritzky, A. R.; Chang, H.-X.; Yang, B. Synthesis 1995, 503. (b) Pasqua, A. E.; Matheson, M.; Sewell, A. L.; Marquez, R. Org. Process Res. Dev. 2011, 15, 467.

(17) Hanna, I.; Ricard, L. Org. Lett. 2000, 2, 2651.

(18) The 3:1 mixture of regioisomers of enol ether $\mathbf{2 4}$ was separated by flash chromatography at this point. Both regioisomers could be carried through the synthesis, but for clarity, only the sequence with the major isomer is reported herein.

(19) Guindon, Y.; Yoakim, C.; Morton, H. E. Tetrahedron Lett. 1983, 24, 2969.

(20) Debenzylation by catalytic hydrogenation gave competitive nitrile reduction to the corresponding amine. 
effected using Guindon's reagent $\left(\mathrm{Me}_{2} \mathrm{BBr}\right) .{ }^{19,20}$ This way, the endocyclic alkene was preserved and could be used to incorporate the additional five-membered ring found in the skeleton of the targeted classes of alkaloids (Scheme 1). After a Swern oxidation ${ }^{21}$ of alcohol 25 and olefination of aldehyde 26 using Still-Gennari conditions, ${ }^{22}$ we were able to obtain the key step precursor 3 (Scheme 4). ${ }^{23}$

Upon treatment with triflic anhydride, formamide $\mathbf{3}$ was activated and trapped intramolecularly to generate the iminium intermediate 29 that was cleanly formed at rt within only 5 min, as seen by ${ }^{1} \mathrm{H}$ NMR spectroscopy (Scheme 6). ${ }^{24}$ When diisopropylethylamine was added, ylide $\mathbf{2}$ was generated and trapped intramolecularly to furnish the tetracyclic adduct 1 in $44 \%$ yield, again at $\mathrm{rt}$ and in only 15 min. ${ }^{25,26}$ From a molecule containing only two stereocenters, we were able to generate four new stereocenters in a fully controlled manner, along with three $\mathrm{C}-\mathrm{C}$ bonds and three new cycles in one pot within $20 \mathrm{~min}$ at rt. The required temperature and time for the dipolar cycloaddition of ylide 2 contrast with the conditions necessary for Coldham's cascade precursor 11, even though the ylide and the dipolarophile are identical. In fact, our more elaborated substrate 3 contains a seven-membered ring that freezes the dipolarophile chain in the axial orientation necessary for the cycloaddition, whereas this reactive conformation is not the preferred one with $\mathbf{1 1}$.

In conclusion, we synthesized the tetracyclic core of daphnilactone B-type and yuzurimine-type alkaloids in only 18 steps from commercially available compound $\mathbf{1 3}$. Our approach constitutes the first example of the con-

(21) Among all of the oxidation conditions tested, only the Swern conditions were tolerated by the silyl enol ether group. For the Swern oxidation, see: Mancuso, A. J.; Huang, S. L.; Swern, D. J. Org. Chem. $\mathbf{1 9 7 8}, 43,2480$.

(22) Still, W. C.; Gennari, C. Tetrahedron Lett. 1983, 24, 4405.

(23) The modest yield for the oxidation and olefination is attributable to the relative sensibility of the silyl enol ether towards aqueous workup and purification on silica gel.

(24) See the Supporting Information.

(25) $\alpha-C N$ 1a: $\beta$-CN $\mathbf{1 b}=1: 1.1$. Upon reduction, both diastereomers will lead to the same product.

(26) Although the key step averages a $76 \%$ yield per $\mathrm{C}-\mathrm{C}$ bond, the moderate yield, compared to previous model studies (see ref 8), could be partly explained by the generation for the first time of a challenging quaternary center through the Vilsmeier-Haack cyclization.
Scheme 6. Synthesis of the Tetracyclic Core of Daphnilactone B-Type and Yuzurimine-Type Alkaloids
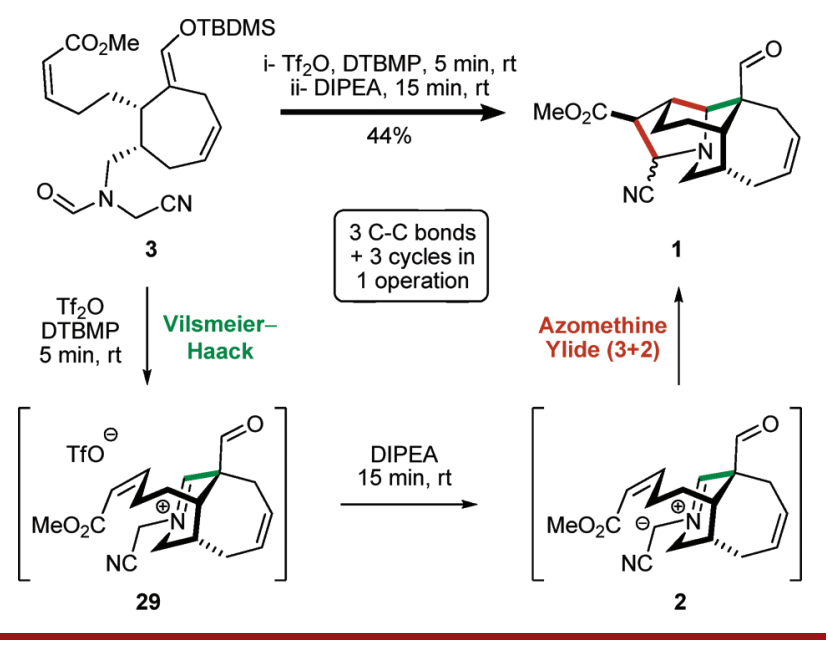

struction of the core of these alkaloid families bearing the seven-membered ring, which is properly functionalized for further incorporation of the required cyclopentene ring found in the natural products. It also demonstrates the power of the intramolecular Vilsmeier-Haack and azomethine ylide cycloaddition sequence as an efficient strategy to rapidly build molecular complexity in applications to the total synthesis of highly challenging natural products. The completed syntheses of alkaloids in the two targeted classes will be reported in due course.

Acknowledgment. This research was supported by the Natural Science and Engineering Research Council (NSERC) of Canada, the Canadian Fund for Innovation (CFI), and the Université de Sherbrooke. FQRNT, NSERC, and CGCC doctoral fellowships to J.B. and Université de Sherbrooke doctoral fellowships to J.B. and F.L are also gratefully acknowledged.

Supporting Information Available. Experimental procedures, spectral data, and characterization for all relevant compounds. This material is available free of charge via the Internet at http://pubs.acs.org. 